\title{
O Estudo de Colisões através do Som
}

\author{
Marisa Almeida Cavalcante, Elias da Silva, Reginaldo do Prado \\ GoPEF-PUC/SP, Pontifícia Universidade Católica de São Paulo, Departamento de Física \\ Rua Marques de Paranaguá 111, CEP 01306-000, São Paulo, SP \\ marisac@pucsp.br \\ Rafael Hagg \\ Instituto de Física, Universidade Federal do Rio Grande do Sul \\ Caixa Postal 15051, 91501-970, Porto Alegre, RS \\ haag@if.ufrgs.br
}

Recebido em 3 de abril, 2002. Aceito em 29 de abril, 2002.

\begin{abstract}
O experimento proposto neste artigo permite ao professor de Física estabelecer uma metodologia computacional para o estudo de movimentos e, particularmente, do coeficiente de restituição em colisões através do espectro sonoro emitido por impactos sucessivos de uma esfera em uma superfície plana. O método de medida sugerido, além de requerer um equipamento de fácil acesso, propicia adequada precisão, como revelam os dados obtidos, já que a aquisição é realizada através de placas de som de microcomputadores pessoais, com tempos de resolução, hoje, da ordem de $23 \mu$ s para taxas de amostragem de até $44 \mathrm{Khz}$. Utilizamos um software de análise do espectro sonoro que apresenta o sinal na forma de gráfico de freqüência versus tempo, livremente disponível na Internet e com uma ampla disponibilidade de recursos.
\end{abstract}

This article presents an experience that allows determining the restitution coefficient in collisions using spectrum of the sound emitted by successive impacts of a sphere in a plane surface. The data are acquired through PC sound card, with times of resolution of order of $22 \mu$ s and sampling rates of up to $44 \mathrm{KHz}$ and that allows to obtain results with a good precision as it can be seen through the results obtained in this work. The spectrum of the sound is analyzed with shareware software that presents the sign in the graphic form of frequency versus time and that has many resources. The equipment used in this experiment is found easily, as well as the software to analyze the sound sign.

\section{Modelo}

Quando uma esfera é solta de uma determinada altura e colide com uma superfície plana e lisa, possivelmente voltará a subir até uma altura menor que a inicial e novamente colidirá com o piso. Este movimento pode se repetir algumas vezes até o momento em que a esfera não mais deixará o solo e permanecerá em repouso tendo perdido toda energia de movimento. Denominamos de coeficiente de restituição, $\varepsilon$, a relação entre os módulos das velocidades antes e após um impacto. Uma colisão elástica é caracterizada por $\varepsilon=1$,

$$
\frac{m_{2}}{m_{1}} \cong 0
$$

diferentemente uma colisão completamente inelástica possuirá $\varepsilon=0$. Um modelo simplificado[1], mas que eficientemente pode ser usado para representar a colisão entre dois corpos está visto na Fig. 1. Usando
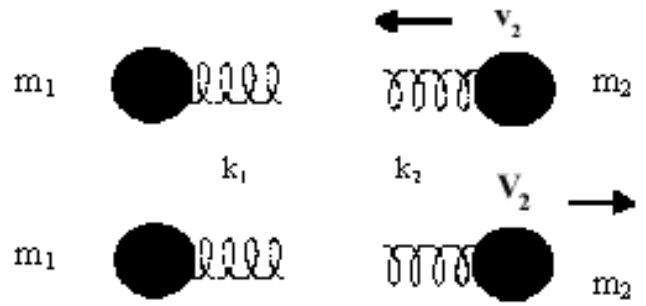

$k_{1} \frac{m_{1}}{m_{1}} \cong 0 \quad k_{2}$

Figura 1. Um modelo de colisão entre uma massa $m_{1}$ de constante elástica $k_{1}$ e uma massa $m_{2}$ de constante elástica $k_{2}$. As velocidades imediatamente antes, $\left(v_{2}\right)$ e após $\left(V_{2}\right)$ a colisão estão mostradas acima.

esta figura para descrever nosso experimento, as massas $m_{1}$ e $m_{2}$ representam a superfície e a esfera respectivamente. A velocidade da esfera imediatamente antes do 
impacto é dada por $v_{2}$ e, logo após perder o contato com a superfície, será $V_{2}$. Assumimos, no experimento que a massa $m_{1}$ possui um valor muito grande se comparada à massa da esfera, $m_{2}$. Usando-se as leis de conservação do momentum linear, é possível verificar que a massa $m_{1}$ permanece em repouso após o choque com a massa $m_{2}$, o que está de acordo com o senso comum. Neste modelo de colisão, a deformação que a superfície e/ou esfera sofre em virtude do impacto pode ser mais bem entendida acoplando-se molas de constantes elásticas $k_{1}$ e $k_{2}$ na superfície e na esfera, respectivamente.

Durante o impacto, exatamente no instante em que a velocidade da esfera torna-se nula, se admitirmos que não há perda de energia até este momento (uma boa aproximação), logo toda a energia cinética existente antes da colisão será convertida em energia potencial armazenada nas molas. Se a totalidade dessa energia fosse novamente transformada em energia de movimento $(\varepsilon$ $=1$ ), veríamos a esfera retornar até a altura em que foi solta (admitindo um movimento unidimensional). Na realidade isto não ocorre, pois parte ou a totalidade (colisão completamente inelástica) desta energia é perdida. O coeficiente de restituição está intimamente relacionado com a energia consumida durante essa efêmera deformação e depende da combinação dos valores de $k_{1}$ e $k_{2}$ [2]. Explorando este modelo de colisão com mais atenção, observamos que, quando uma esfera colide com uma superfície muito rígida $\left(k_{1}>>k_{2}\right)$, apenas a esfera sofrerá deformação durante o impacto. Às vezes esta deformação pode ser irreversível. Isso ocorre quando uma esfera com grande energia cinética inicial, $E_{i}$, e $k$ elevado, colide com uma superfície cujo coeficiente de deformação, $k$, facilmente passa do regime elástico para o plástico ${ }^{1}$, por exemplo, certos tipos de madeira. Se observarmos com atenção, notaremos que as colisões nas condições descritas acima produzem pequenas "marcas" na madeira e esta energia gasta na deformação provoca uma redução do valor numérico do coeficiente de restituição. As dimensões da superfície e da esfera também afetam o coeficiente de restituição, particularmente se a duração do impulso for comparável com o período de vibração dos objetos envolvidos no impacto [3]. Outra complicação no estudo da dinâmica da colisão reside no fato de que $k_{1}$ e $k_{2}$ não são constantes durante o choque e, além disto, possuem comportamento não-linear [1]-[2]. Por isso, é muito complexo descrever os processos envolvidos na perda de energia durante a colisão [4]. Apesar disto - felizmente - podemos extrair várias informações relevantes para o estudo de colisões entre a esfera e a superfície, apenas analisando o sinal sonoro produzido durante os impactos.

\section{Introdução}

Nossa proposta utiliza recursos computacionais para a coleta de dados, que dispensa o uso de interfaces conversoras externas, recorrendo às específicas como a placa de som que, normalmente, acompanha os microcomputadores atuais. Tal procedimento nos permite criar novas perspectivas para a inserção da experimentação assistida por computador no ensino de Ciências, visto que promove facilidades como:

- custo zero para a implementação de sistemas de medida;

- afasta a necessidade de conhecimentos em eletrônica para a construção de interfaces conversoras;

- não é necessário dominar nenhuma linguagem de programação para iniciar um trabalho nesta área.

Trabalhos nacionais recentes, Haag [5], Montarroyos [6] e Aguiar [7], apontam grandes possibilidades de renovação nas técnicas em medidas físicas para um laboratório didático.

Dentre as inúmeras possibilidades, optamos por utilizar a placa de som de um PC para a coleta de dados, por várias razões:

a) simplicidade nos equipamentos necessários para desenvolver o experimento;

b) alta precisão nos resultados obtidos, já que os tempos de resolução na maioria das placas de som dos PCs podem ser tão pequenos quanto $23 \mu$ seg (para taxas de amostragem de $44 \mathrm{KHz}$ ).

Existem vários softwares livres disponíveis na Internet [8] que permitem usar as entradas e saídas de áudio da placa de som para simular e, também, transformar o microcomputador em instrumento de medida, com um excelente desempenho. Neste trabalho utilizaremos, para obtenção e análise de espectro sonoro, o Spectrogram [9], que apresenta o sinal na forma de gráfico de freqüência versus tempo.

A proposta consiste em obter o registro em um arquivo wav do som emitido, nos impactos sucessivos de uma esfera solta de uma altura h em uma superfície plana.

Durante o impacto da esfera com a superfície de apoio, um som é irradiado, cujo registro é efetuado através de um microfone conectado à entrada da placa de som do PC. A partir do sinal sonoro, faz-se uma reprodução gráfica do tempo obtido entre colisões sucessivas e com isso várias informações, tanto cinemáticas quanto dinâmicas podem ser facilmente obtidas.

\footnotetext{
${ }^{1}$ Caracterizamos por regime elástico um sistema que admite expansão/compressão reversível, em que o valor da constante elástica, $k$, permanece constante em função da força que lhe é aplicada. Se a força aplicada ultrapassar o limite admitido de expansão/compressão, o sistema passa para o regime plástico em que $k$ não mais será constante, e a deformação provocada por esta força será irreversível.
} 


\section{Determinação do Coeficiente de Restituição}

O trabalho recentemente publicado por I. Stensgaard e E. Laegsggard [10] descreve a técnica de medida que utilizaremos para obter informações sobre o coeficiente de restituição na interação entre uma esfera e uma superfície horizontal.
O princípio deste método, que pode ser encontrado em detalhes no artigo de Alan Bernstein [11] publicado em 1977 e depois atualizado por P.A Smith, C.D Spencer, e D. E. Jones [12] na década de 80, consiste basicamente no registro, através de um microfone, do som produzido pelos impactos sucessivos de uma esfera, solta de uma altura $H_{1}$ contra uma superfície plana, conforme indica o esquema abaixo.

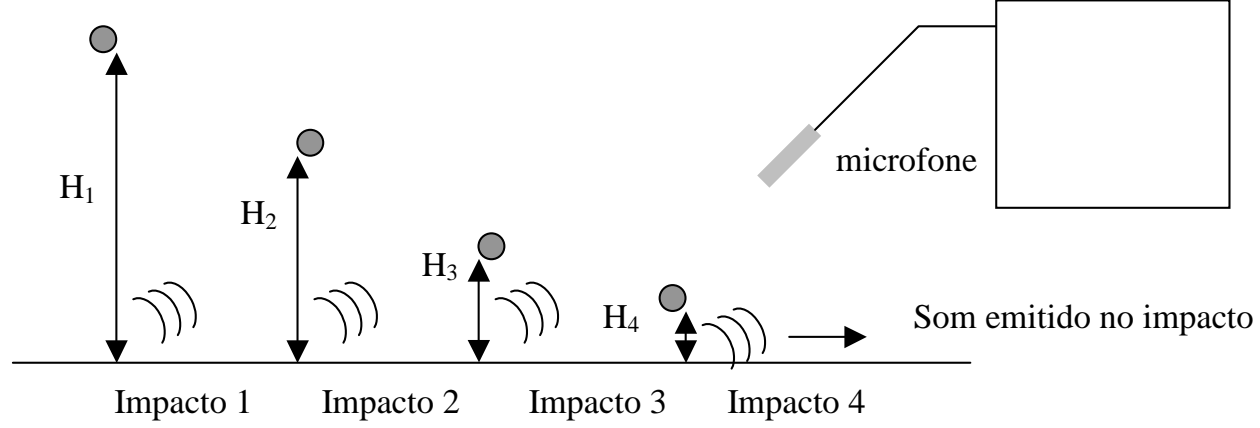

Figura 2. Diagrama do experimento.

\section{freqüência}

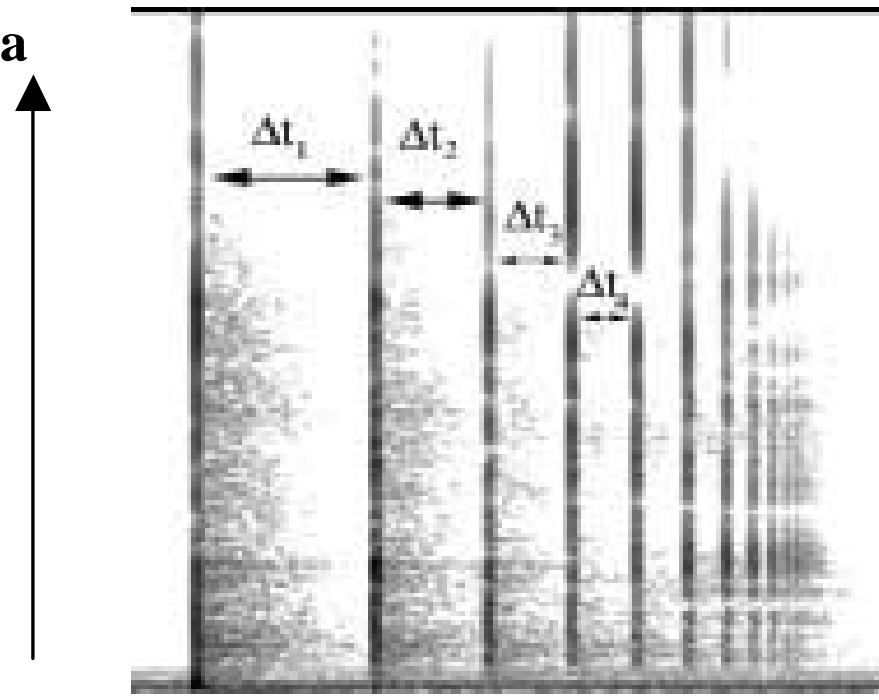

\section{tempo}

Figura 3. Representação visual do sinal sonoro obtido através do software Spectrogram.

Os intervalos de tempo entre os impactos sucessivos podem ser facilmente obtidos diretamente pelo observador, conforme indica a Fig. 3 .

A cada impacto da esfera contra a superfície, ocorre a perda de energia cinética, reduzindo-se a altura máxima que ela pode atingir no seu retorno $\left(H_{n+1}<\right.$ $\left.H_{n}\right)$. A grandeza que determina esta fração de perda é o coeficiente de restituição $\varepsilon$, que pode ser determinado através da relação entre as velocidades, depois e antes da colisão. 
Torna-se muito útil definirmos um fator de perda de energia cinética, $f$, após a colisão entre a esfera e o piso.

$$
\left.f=\left(E_{i}-E_{f}\right) / E_{i}=\left(\frac{1}{2} m_{2} v_{2}^{2}-\frac{1}{2} m 2 V_{2}^{2}\right)\right) / \frac{1}{2} m_{2} v_{2}^{2}=v_{2}^{2}-V_{2}^{2} / v_{2}^{2}
$$

onde Ei indica a energia cinética inicial, antes da colisão, e Ef a energia cinética após a colisão. Lembrandose que $\varepsilon=V_{2} / v_{2}$, então, $\mathrm{f}$, torna-se:

$$
f=\left(1-\varepsilon^{2}\right)
$$

Numa colisão perfeitamente elástica, temos $\varepsilon=1$, $\operatorname{assim} f=0$, ou seja, nenhuma energia cinética será perdida durante a colisão. Nesta situação teórica, podemos determinar facilmente o comportamento da esfera que está colidindo com o piso. Encontramos as equações que descrevem o movimento da esfera com algumas poucas manipulações algébricas, e os resultados mostram que a esfera, após a colisão, voltará até o ponto de onde foi solta. Este resultado jamais será visualizado, pois sempre a colisão entre a esfera e a superfície será acompanhada de uma perda de energia fazendo com que a altura máxima alcançada pela esfera após o impacto seja sempre menor que a altura inicial. É fácil notar que quanto menor o coeficiente restituição na colisão esfera-superfície maior é a taxa de redução nos intervalos de tempo entre os impactos. Esta dependência pode ser vista no gráfico da Fig. 4 para duas interações distintas, esfera de vidro colidindo com superfície de madeira e uma superfície de pedra.

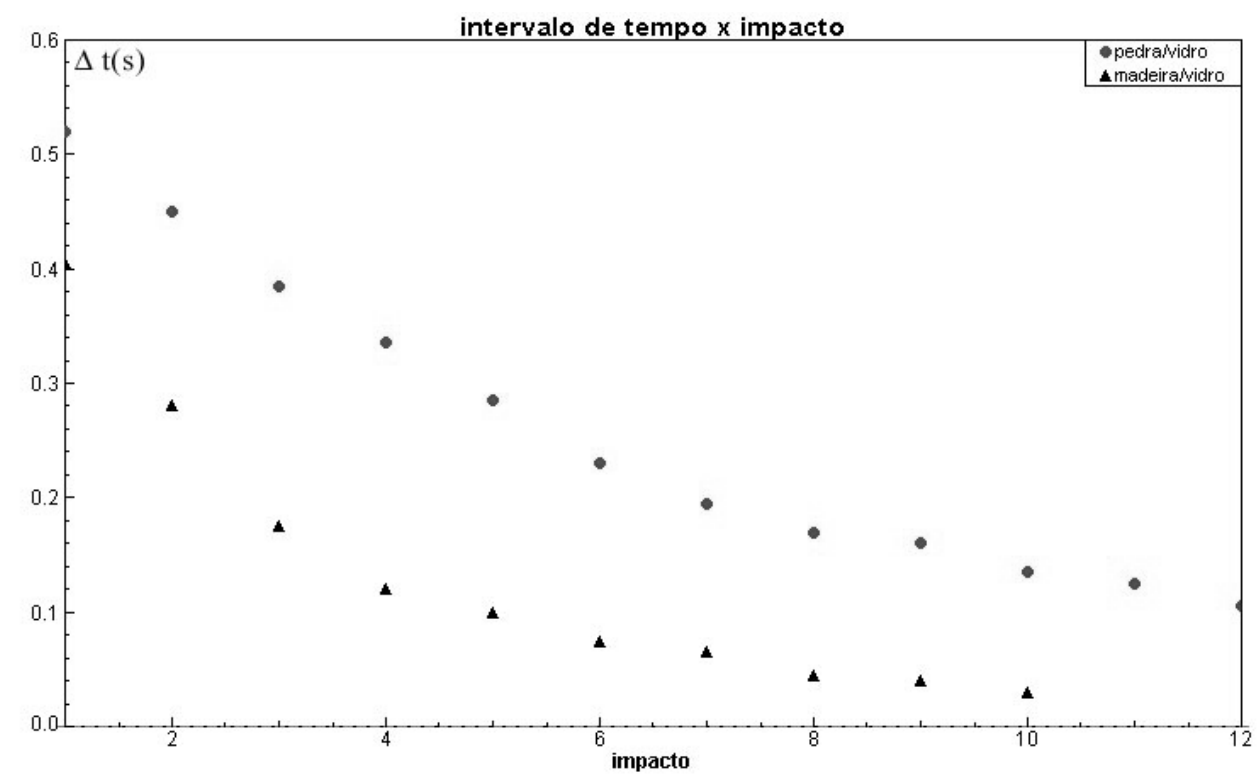

Figura 4. O gráfico mostra a taxa com a qual o intervalo de tempo entre colisões sucessivas diminui para dois tipos de interações distintas, indicando que, para a interação vidro x pedra, o coeficiente de restituição apresenta um maior valor.

Para uma interação esfera - superfície, a relação entre o valor da componente vertical das velocidades antes e depois do impacto fornece o valor do coeficiente de restituição.

Supondo que a fração de perda de energia cinética é constante, independendo do valor da velocidade de impacto da esfera de massa $m_{2}$, teremos que:

$$
\varepsilon=\frac{v_{n+1}}{v_{n}}
$$

onde $n$ representa o índice associado ao impacto.

Para o impacto de ordem $n$, o valor da velocidade pode ser obtido pela relação:

$v_{n}=\frac{q \Delta t_{n}}{2}$, onde $\Delta t_{n}$ é o intervalo de tempo entre impactos sucessivos.

Substituindo os valores das componentes verticais de velocidade na equação 2 , teremos;

$$
\varepsilon=\frac{\Delta t_{n+1}}{\Delta t_{n}}
$$

onde $n$ representa o índice associado ao impacto.

Propomos, neste artigo, que o coeficiente de restituição, diferentemente dos trabalhos anteriormente publicados [10]-[12] seja obtido através da inclinação 
da reta no gráfico $\Delta t_{n+1} \times \Delta t_{n}$. Com o objetivo de determinar uma média, para o coeficiente de restituição, vários lançamentos foram efetuados de diferentes alturas $(\mathrm{h}=100,75,50$ e $25 \mathrm{~cm})$ para cada tipo de in- teração.

Os gráficos abaixo mostram alguns resultados obtidos:

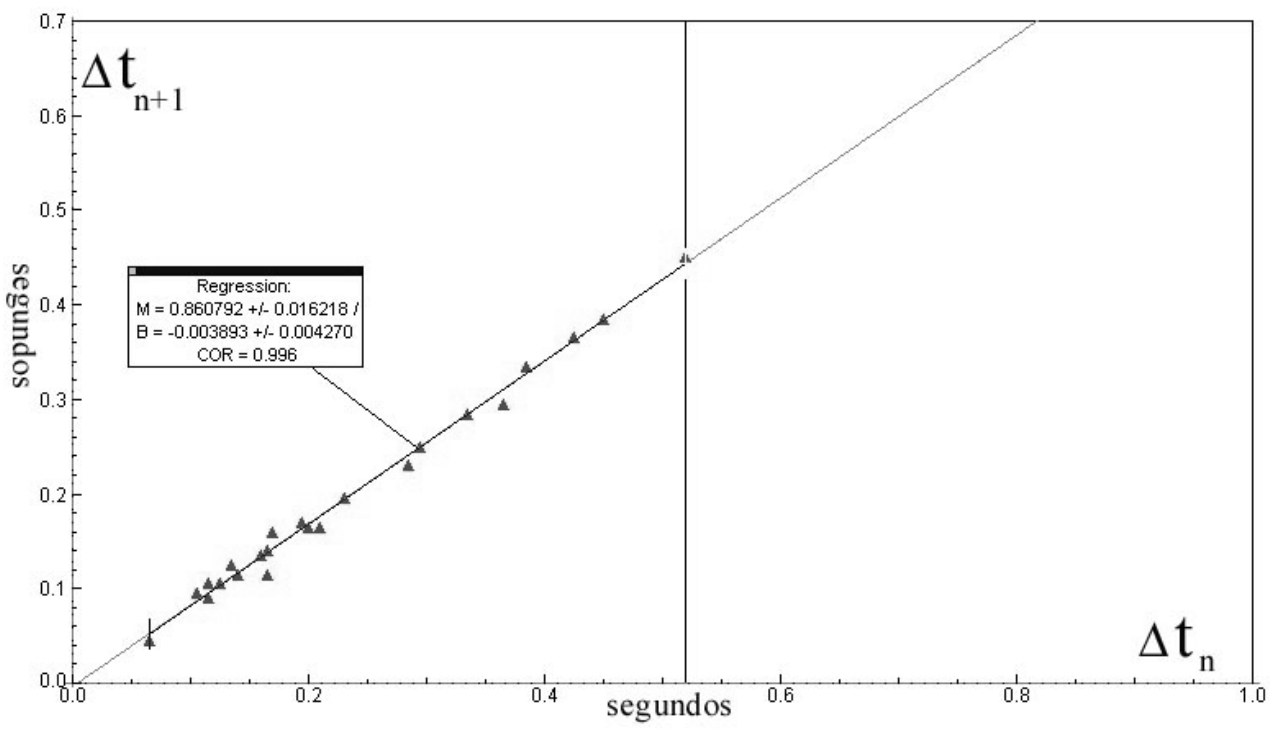

Figura 5. Interação vidro $\times$ pedra, coeficiente de restituição $e=0,86$.

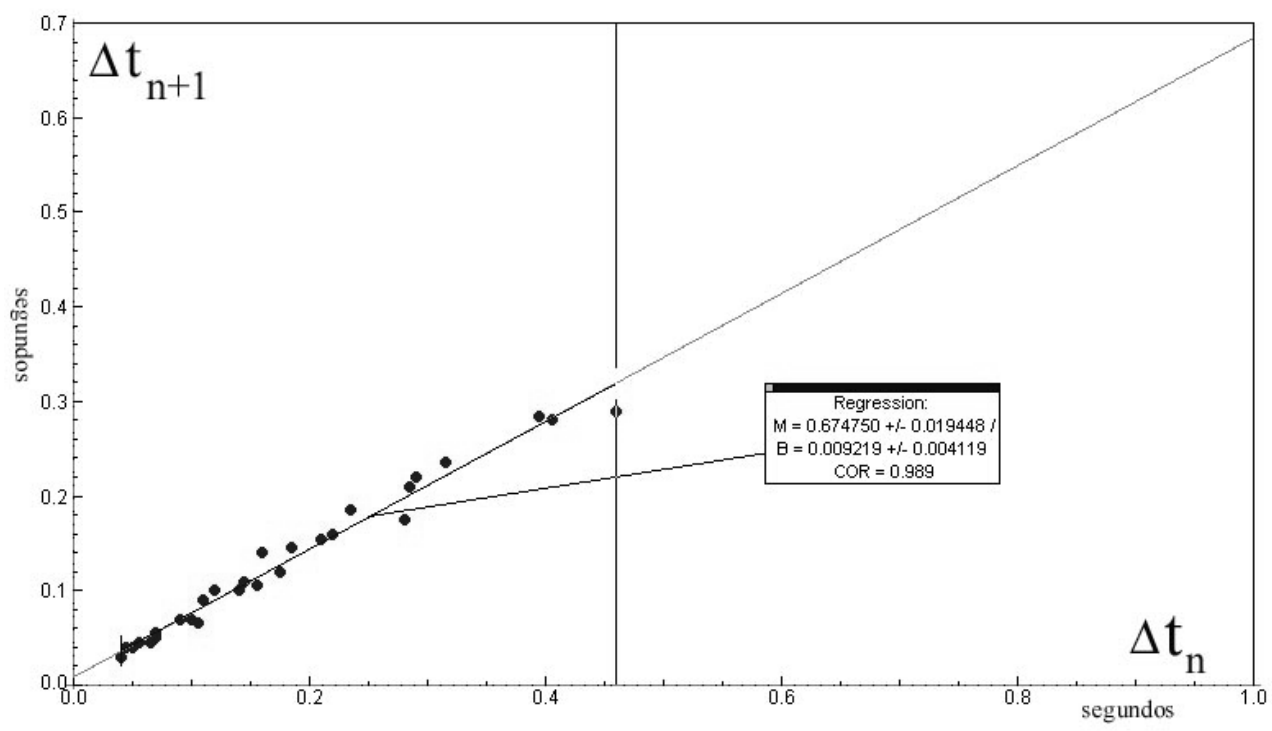

Figura 6. Interação vidro $\times$ madeira, coeficiente de restituição $e=0,67$. 


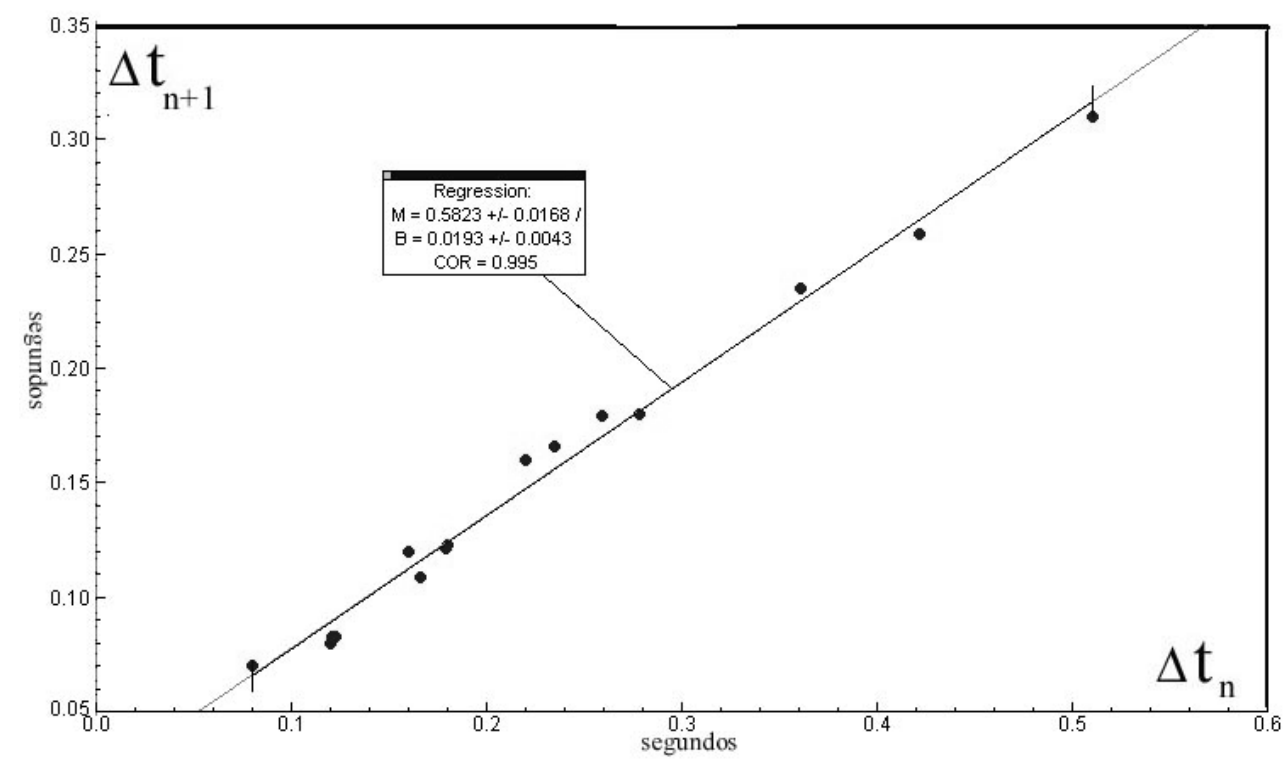

Figura 7. Interação aço $\times$ madeira, coeficiente de restituição $e=0,58$.

\section{Determinação das alturas de lançamento}

Supondo que a fração de perda de energia cinética, $f$, é constante, para uma dada interação, podemos determinar a altura da qual uma esfera foi abandonada em relação à superfície.

Para compreender de que maneira podemos determinar a altura de lançamento da esfera, considere-se $\Delta t_{n, m}$ como o intervalo de tempo para um dado impacto $\mathrm{n}$ e lançamento $m$. A componente vertical de velocidade de uma esfera que alcança a altura $H_{2}$ da Fig. 2 é dada pela relação:

$$
v_{\text {depois }}=\frac{g \Delta t_{1,1}}{2}
$$

onde $\Delta t_{1,1}$ representa o do intervalo de tempo entre o primeiro e segundo impacto para o lançamento 1 . A velocidade com a qual a esfera atinge o solo é

$$
v_{\text {antes }}=\sqrt{2 g h_{\text {inicial }}} \text {. }
$$

Por outro lado, o coeficiente de restituição é dado por:

$$
\frac{v_{\text {depois }}}{v_{\text {antes }}}=\varepsilon .
$$

Generalizando, para qualquer lançamento $m$ e impacto $n$, a altura atingida comparada ao lançamento 1 , cuja altura inicial é conhecida, temos:

$$
\frac{\Delta t_{n, 1}}{\sqrt{H_{\text {inicial }, 1}}}=\frac{\Delta t_{n, m}}{\sqrt{H_{\text {inicial }, m}}}=\varepsilon
$$

$$
\frac{\Delta t_{n, 1}}{\Delta t_{n, m}}=\sqrt{\frac{H_{\text {inicial }, 1}}{H_{\text {inicial }, m}}} .
$$

Assim, podemos obter facilmente a relação entre as alturas iniciais de diferentes lançamentos.

Os gráficos abaixo mostram claramente a relação linear, cujo valor de inclinação fornece a raiz quadrada da relação entre as alturas iniciais estabelecidas em cada caso.

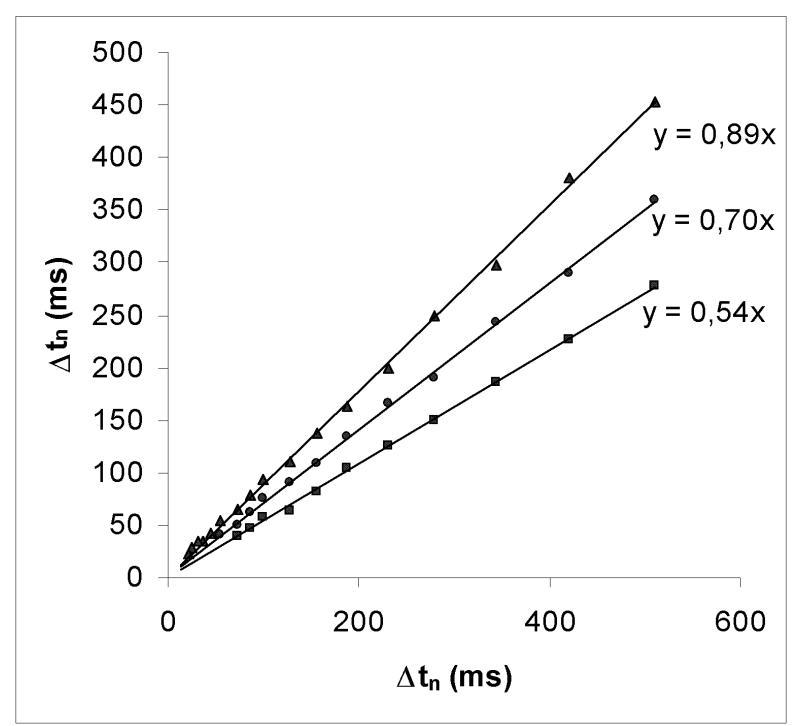

Figura 8. Fixamos um lançamento de altura inicial conhecida para o eixo $\mathrm{x}(\mathrm{H}=100 \mathrm{~cm})$ e para o eixo $\mathrm{y}$ inserimos os valores dos intervalos de tempo dos impactos correspondentes às alturas que consideramos desconhecidas.

A partir da inclinação das retas obtidas da eq. (9), tomando-se $H_{\text {inicial }, 1}=100 \mathrm{~cm}$, obtêm-se os resultados 
da tabela 1

Tabela 1

\begin{tabular}{|c|c|c|c|}
\hline Inclinação da reta & 0,54 & 0,70 & 0,89 \\
\hline Altura obtida em cm & 29,2 & 49,0 & 79,2 \\
\hline
\end{tabular}

Os valores fixados para estas alturas foram $30 \mathrm{~cm}$, $50 \mathrm{~cm}$ e $80 \mathrm{~cm}$ aproximadamente, em boa concordância com os valores obtidos.

\section{Determinação da aceleração da gravidade}

Ao considerarmos constante a fração de perda de energia cinética da esfera nos impactos sucessivos de uma esfera com uma dada superfície, estabelecemos as condições de contorno necessárias para determinar o valor da aceleração da gravidade.

Para compreender de que maneira podemos determinar o valor da aceleração da gravidade, considere-se que o valor da velocidade vertical da esfera solta a altura h, antes da colisão com uma superfície plana, é dada por:

$$
v_{\text {antes }}=\sqrt{2 g h}
$$

Por outro lado, o valor da componente vertical da velocidade depois da colisão é dada por:

$$
v_{\text {depois }}=\frac{g \Delta t_{1}}{2}
$$

Assim temos que:

$$
\varepsilon^{2}=\frac{g \Delta t_{1}^{2}}{8 h}
$$

Substituindo-se o valor de $\varepsilon$ pela relação entre os intervalos de tempo, indicado na equação 3, temos;

$$
g=\frac{8 h \Delta t_{2}^{2}}{\Delta t_{1}^{4}}
$$

Sendo h, a altura de que a esfera é solta, $\Delta t_{2}$ o intervalo de tempo obtido entre o impacto 3 e 2 da Fig. 2 e $\Delta t_{1}$, e intervalo de tempo obtido entre o impacto 2 e 1 (Fig. 2).

Se consideremos constante o valor do coeficiente de restituição, a relação da Eq. 12 deve ser obedecida, para qualquer interação, o que permite obter o valor da aceleração da gravidade.

Vários lançamentos foram realizados para distintas interações esfera_ superfície, obtendo-se o gráfico representado na Fig. 9.

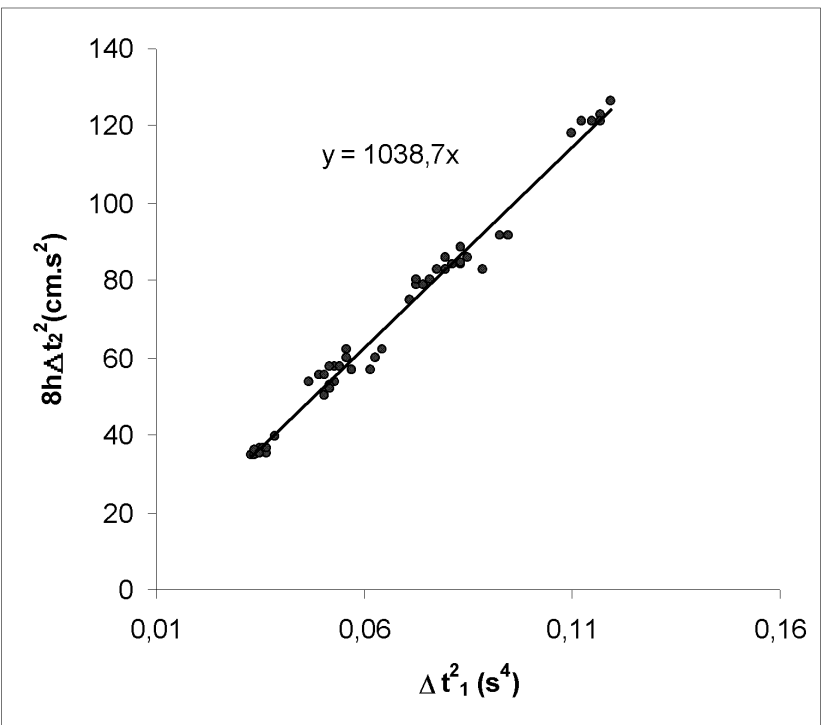

Figura 9. gráfico mostra a relação linear obtida para vários lançamentos com diferentes pares superfície $\times$ esfera. $O$ valor da inclinação da reta fornece a aceleração da gravidade.

O valor obtido para a aceleração da gravidade, considerando-se a propagação de erros sistemáticos e estatísticos [15], foi de $(1038,7 \pm 49,6) \mathrm{cm} / \mathrm{s}^{2}$, valor este bastante satisfatório.

\section{Conclusão}

Neste artigo mostramos um modo experimental para obter alguns parâmetros físicos envolvidos numa colisão entre esferas e superfícies de diferentes materiais, usando o espectro sonoro irradiado durante a colisão. Os resultados obtidos revelam que a precisão do experimento é adequada e que ele pode ser facilmente implementado, praticamente sem custos, em qualquer laboratório didático de física que possua um $\mathrm{PC}$ com placa de som.

O valor obtido para a aceleração da gravidade permite assegurar, com um grau de segurança considerável, a aplicabilidade deste método, já que o valor esperado está contemplado no intervalo obtido.

A inviabilidade deste método está associada à garantia experimental de manter constante a fração de perda de energia cinética nos impactos sucessivos. Assim, desaconselhamos que se proceda a interações em que deformações no impacto são fortemente evidenciadas, por exemplo, colisões com superfícies de madeira.

Quanto maior o valor para o coeficiente de restituição melhor será a convergência dos resultados e menor devem ser os desvios experimentais obtidos.

O professor interessado pode ir além e sugerir aos alunos comparar os dados obtidos no experimento com os previstos através de processos de modelação computacional [13] que facilmente são executados com o auxílio de softwares específicos para esta tarefa, como; Modellus, Matlab [14] entre outros. 


\section{Referências}

[1] CROSS, R. The bounce of a ball. Am. J. Phys. 67 (4), March 1999, pp. $222-227$.

[2] CROSS, R. The coefficient of restitution for collisions of happy balls, unhappy balls, and tennis balls. Am. J. Phys. 68 (12), November 2000, pp. 1025 - 1031.

[3] KHARAZ, A. H., GORHAM, D. A. AND SALMAN, A. D., An experimental study of the elastic rebound of spheres. Powder Technology, 120, 2001, pp. $281-291$.

[4] GUGAN, D. Inelastic collision and the Hertz theory of impact. Am. J. Phys.

68 (11), October 2000, pp. 920 - 924.

[5] HAAG, R. Utilizando a Placa de Som do Micro PC no Laboratório didático de Física; Rev. Bras. de Ensino de Física, Vol. 23, no. 2, Junho de 2001, pp176- 183.

[6] MONTARROYS, E. e MAGNO, W. C. Aquisiçẫo de Dados com a Placa de Som do Computador. Revista Brasileira de Ensino de Física, Vol. 23, no. 1, Março de 2001, pp. 57-62.

[7] http://www.if.ufrj.br/ carlos/ensino.html último acesso em abril de 2002.
[8] http://www.if.ufrgs.br/tex/fis01043 último acesso em dezembro de 2001

[9] http://www.monumental.com/rshorne/gram.html último acesso em dezembro de 2001

[10] STESGAARD, I. and LAEGSGAARD, E. Listening to the coefficient of restitution-revisited. Am. J. Phys. 69 (4), March 2001, pp.301- 305.

[11] BERNSTEIN, A. D. Listening to the coefficient of restitution. Am. J. Phys., 45 (1), January 1977, pp.4143.

[12] SMiTh, A P., SPENCER, D. C. and JONES, E. D. Microcomputer listen to the coefficient of restitution. Am. J. Phys., 49 (3), Feb1981, pp.136- 40.

[13] VEIT, E. A. e TEODORO, V. - trabalho submetido à Revista Brasileira de Ensino de Física.

[14] http:// www.mathworks.com. último acesso em dezembro de 2001

[15] VUOLO, H. J Fundamentos da Teoria de Erros Editora Edgar Blucher LTDA 2.a edição -1996. 